\title{
Gastric antral vascular ectasia in cirrhotic patients: absence of relation with portal hypertension
}

\author{
L Spahr, J-P Villeneuve, M-P Dufresne, D Tassé, B Bui, B Willems, D Fenyves, \\ G Pomier-Layrargues
}

\begin{abstract}
Background-Portal hypertensive gastropathy and gastric antral vascular ectasia (GAVE) are increasingly recognised as separate entities. The pathogenic role of portal hypertension for the development of GAVE is still controversial.
\end{abstract}

Aims-To evaluate the effects of portal decompression on chronic bleeding related to GAVE in cirrhotic patients.

Methods-Eight patients with cirrhosis and chronic blood loss related to GAVE were included. GAVE was defined endoscopically and histologically.

Results-All patients had severe portal hypertension (mean portocaval gradient (PCG) $26 \mathrm{~mm} \mathrm{Hg}$ ) and chronic low grade bleeding. Seven patients underwent transjugular intrahepatic portosystemic shunt (TIPS) and one had an end to side portacaval shunt. Rebleeding occurred in seven patients. In these, TIPS was found to be occluded after 15 days in one patient; in the other six, the shunt was patent and the PCG was below $12 \mathrm{~mm} \mathrm{Hg}$ in five. In the responder, PCG was $16 \mathrm{~mm} \mathrm{Hg}$. Antrectomy was performed in four nonresponders; surgery was uneventful, and they did not rebleed after surgery, but two died 11 and 30 days postoperatively from multiorgan failure. In one patient, TIPS did not control GAVE related bleeding despite a notable decrease in PCG. This patient underwent liver transplantation 14 months after TIPS; two months after transplantation, bleeding had stopped and the endoscopic appearance of the antrum had normalised.

Conclusions-Results suggest that GAVE is not directly related to portal hypertension, but is influenced by the presence of liver dysfunction. Antrectomy is a therapeutic option when chronic bleeding becomes a significant problem but carries a risk of postoperative mortality. (Gut 1999;44:739-742)

Keywords: gastric antral vascular ectasia; portosystemic shunt; portal hypertension; cirrhosis; portacaval shunt

Correspondence to: Dr G Pomier-Layrargues, Liver Unit, Centre de recherche du CHUM, Campus Saint-Luc, 264, East René-Lévesque Blvd, Montréal, Québec, Canada $\mathrm{H} 2 \mathrm{X} 1 \mathrm{P} 1$.

Accepted for publication 18 November 1998

Gastric mucosal lesions are being increasingly recognised as common features in cirrhotic patients. ${ }^{1}$ It has been suggested that portal hypertensive gastropathy (PHG) and gastric antral vascular ectasia (GAVE) are two distinct entities in cirrhotic patients. ${ }^{2}$
These lesions can be differentiated by endoscopic and histological findings. PHG is always associated with cirrhosis and is observed mostly in the fundus and corpus of the stomach; musocal red spots and the so called "mosaic pattern" are present and histological examination only shows vascular ectasia in the mucosa. By contrast GAVE can occur in both cirrhotic and non-cirrhotic patients. Lesions are found almost exclusively in the antrum and red spots are either aggregated in linear stripes (as in the "watermelon stomach") or diffusely spread. Histologically, vascular ectasia are present in the mucosa and are associated with fibrin thrombi, fibrohyalinosis, and spindle cell proliferation. $^{2}$

Occult or overt bleeding occurs more frequently as a complication of GAVE than of PHG. Multiple transfusions may be required during follow up. It has been suggested previously that prevention of rebleeding from gastric mucosal lesions in cirrhotic patients can be achieved by decreasing portal pressure either pharmacologically using $\beta$ blockers or surgically with portocaval shunt. ${ }^{3-5}$ In the present study, we evaluated the usefulness of portal decompression in the treatment of chronic bleeding from GAVE and assessed the potential contribution of portal hypertension to these lesions.

\section{Patients and methods}

Inclusion criteria were as follows: biopsy proven cirrhosis; GAVE as shown by typical endoscopic and histological findings as described above; and chronic recurrent bleeding unresponsive to $\beta$ blockers.

Eight patients (six men, two women; mean age 65 years) were evaluated (table 1). Cirrhosis was related to chronic alcoholism in four patients, to primary biliary cirrhosis (PBC) in one, was cryptogenic in two, and was due to hepatitis B virus infection in one. One patient belonged to Pugh class A and seven to Pugh class B. Oesophageal varices were also present in five patients (large varices in two, small varices in three); in these patients, there was no evidence of active bleeding from varices whereas antral oozing was observed at endoscopy. Chronic bleeding caused severe anemia, as shown by a mean lowest haemoglobin level of $65 \mathrm{~g} / 1$ during the observation period before treatment.
Abbreviations used in this paper: GAVE, gastric antral vascular ectasia; PHG, portal hypertensive gastropathy; TIPS, transjugular intrahepatic portosystemic shunt. 
Table 1 Clinical characteristics of the study group

\begin{tabular}{llllllll}
\hline Patient no & Age (years) & Sex & Diagnosis & Pugh's score & Lowest Hb level (g/l) & Previous sclerotherapy & Oesophageal varices \\
\hline 1 & 61 & M & PBC & 9 & 93 & Yes & Grade 3/4 \\
2 & 55 & M & Cryptogenic & 9 & 48 & No & Grade $3 / 4$ \\
3 & 58 & M & Alcoholic & 9 & 69 & No & Grade $1 / 4$ \\
4 & 73 & M & Alcoholic & 5 & 70 & No & Grade $2 / 4$ \\
5 & 75 & F & Cryptogenic & 8 & 53 & No & None \\
6 & 73 & F & B virus & 9 & 53 & Yes & None \\
7 & 65 & M & Alcoholic & 9 & 62 & Yes & None \\
8 & 65 & M & Alcoholic & 9 & 69 & & \\
\hline
\end{tabular}

PBC, primary biliary cirrhosis; Hb, haemoglobin.

Table 2 Blood transfusion requirement before and after TIPS

\begin{tabular}{lll}
\hline Patient no & $\begin{array}{l}\text { Before TIPS } \\
\text { (period of time) }\end{array}$ & $\begin{array}{l}\text { After TIPS } \\
\text { (period of time) }\end{array}$ \\
\hline 1 & 12 units (12 months) & 13 units (14 months) \\
$2^{\star}$ & 10 units (1.5 month) & 110 units (5 years) \\
3 & 13 units (5 months) & 0 unit (4 years) \\
4 & 20 units (2 years) & 15 units (12 months) \\
5 & 16 units (2 months) & 17 units (2 months) \\
6 & 10 units (2 months) & 15 units (1 month) \\
7 & 6 units (4 months) & 12 units (18 months) \\
8 & 8 units (4 months) & 6 units (1.5 month) \\
\hline
\end{tabular}

^Patient 2 underwent portocaval shunt.

Seven patients were treated by transjugular intrahepatic portosystemic shunt (TIPS) according to a technique reported previously ${ }^{6}$ and one patient underwent surgical end to side portocaval shunt. The patency of the TIPS (or of the surgical shunt) was assessed every three months by echo Doppler ultrasonography. Shunt catheterisation was performed routinely at two months and one year, and when shunt dysfunction was suspected according to Doppler criteria as reported previously. ${ }^{7}$ When rebleeding occured, usually manifested by anemia secondary to occult bleeding, the source was always documented by endoscopy and shunt catheterisation was performed to measure the portocaval gradient. Shunt dysfunction, defined as an increase in the portacaval gradient above $12 \mathrm{~mm} \mathrm{Hg}$, was treated by shunt dilatation with the addition of a new stent if needed.

\section{Results}

Recurrent bleeding was not controlled by portosystemic shunting in seven patients (TIPS in six and surgical shunt in one). In these patients repeated blood transfusions were needed to maintain haemoglobin levels above $90 \mathrm{~g} / 1$ (table 2) despite adequate portal decompression in five patients (portocaval gradient at rebleeding: 5, 2, 10, 11, $10 \mathrm{~mm} \mathrm{Hg}$; table 3). In one patient, the gradient could not be lowered below 16

Table 3 Correlation between portocaval gradient and rebleeding

\begin{tabular}{lllll}
\hline Patient no & $\begin{array}{l}\text { Portocaval gradient } \\
\text { (mm Hg) before TIPS }\end{array}$ & $\begin{array}{l}\text { Portocaval gradient } \\
(\text { mm Hg) after TIPS }\end{array}$ & Rebleeding & $\begin{array}{l}\text { Portocaval gradient } \\
\text { (mm Hg) at rebleeding }\end{array}$ \\
\hline 1 & 23 & 11 & Yes & 5 \\
$2^{\star}$ & 13 & 1 & Yes & 2 \\
3 & 30 & 16 & No & - \\
4 & 21 & 12 & Yes & 10 \\
5 & 27 & 16 & Yes & 16 \\
6 & 27 & 16 & Yes & Occluded \\
7 & 36 & 15 & Yes & 11 \\
8 & 25 & 11 & Yes & 10 \\
\hline
\end{tabular}

^Patient 2 underwent portocaval shunt. $\mathrm{mm} \mathrm{Hg}$ and in one patient, the TIPS became occluded two weeks after TIPS.

Four of the rebleeders (patients 4, 5, 6, and 8) underwent antrectomy. There were no operative blood losses in the three patients with a patent TIPS before surgery compared with a six blood units loss in the patient with an occluded TIPS. After antrectomy, bleeding did not recur. Two patients died postoperatively (at 11 and 30 days) from multiorgan failure; one patient died from cerebral hemorrhage two years later; and one patient is still alive 10 months after surgery.

One patient (patient 1) experienced continuous occult bleeding despite successful portal decompression by TIPS (gradient: $10 \mathrm{~mm}$ $\mathrm{Hg}$ ). He underwent liver transplantation 14 months after TIPS; bleeding never recurred and the patient was alive eight months after surgery. Endoscopy performed two months after transplantation showed a complete disappearance of antral mucosal lesions.

In the two remaining rebleeders (patients 2 and 7), antrectomy was not considered because of liver failure; these patients died 18 months after TIPS and five years after surgical shunt respectively.

One patient (patient 3) did not experience recurrent bleeding after TIPS. In this patient, endoscopic lesions remained unchanged, but he never required blood transfusion; he is alive five years after the TIPS procedure. The portocaval gradient remained between 16 and $10 \mathrm{~mm} \mathrm{Hg}$ during follow up.

\section{Discussion}

Bleeding from gastric mucosal lesions in patients with portal hypertension is a serious complication. Bleeding is usually slow and insidious and rarely massive and life threatening. ${ }^{89}$ It has been suggested that, in cirrhotic patients, PHG and GAVE are distinct entities on the basis of endoscopic appearance and histological characterisation. ${ }^{2}{ }^{10-12}$

PHG has been found to be associated with portal hypertension; gastric perfusion alterations parallel the severity of $\mathrm{PHG}^{13}$ and it has been suggested that obliteration of oesophageal varices following endoscopic sclerotherapy increases the risk of developing PHG by a back pressure mechanism. ${ }^{14}$

Treatment with $\beta$ blockers has been reported to be successful in controlling bleeding from gastric mucosal lesions in cirrhotic patients. However, in these reports, it is unclear whether patients bled from PHG or from GAVE. ${ }^{34}$ Therefore the true efficacy of $\beta$ blocker therapy cannot be evaluated reliably. However, it is 
worth noting that in our patients, treatment with the non-selective $\beta$ blocker nadolol did not control chronic bleeding from GAVE.

In addition, the results of the present study clearly show that the decrease in portal hypertension following TIPS or shunt surgery was not effective in controlling chronic blood loss related to GAVE. This was particularly obvious in the patient who underwent a surgical shunt; chronic bleeding was never controlled despite a complete normalisation of portal pressure. Similarly, it has been reported that portacaval shunt surgery proved ineffective in treating chronic gastrointestinal blood loss related to GAVE in a patient with portal hypertension as a result of nodular regenerative hyperplasia. ${ }^{15}$

The absence of a relation between the occurrence of GAVE and the presence of portal hypertension is also confirmed by the fact that GAVE has been reported to occur in patients without portal hypertension and without cirrhosis. ${ }^{16}$ Other clinical conditions associated with GAVE include chronic renal failure, scleroderma, bone marrow transplantation, and autoimmune disorders. ${ }^{16-18}$

The pathogenesis of GAVE is unknown. The role of intermittent obstruction of submucosal blood vessels due to repeated mechanical stress related to gastric contractions and leading to acquired vascular ectasia has been proposed by some authors. ${ }^{10}$ It has also been shown that in cirrhosis there is an abnormal antral motor response to a meal in cirrhotic patients with GAVE. ${ }^{19}$ The fibromuscular hyperplasia seen at histology in GAVE supports the hypothesis of repeated mechanical stress induced by gastric peristalsis. The role of hypergastrinaemia in GAVE has been suggested by some authors ${ }^{20}$ but was not confirmed recently. ${ }^{2}$ This hormone acts on both antral and pyloric contractility and may thereby impair gastric emptying and create repeated mucosal lesions such as prolapse through the pylorus. Lowes and Rode ${ }^{21}$ raised the possibility that the dilated blood vessels of GAVE could be related to the local release of vasoactive substances. They showed in a resected specimen of GAVE a notable neuroendocrine cell proliferation containing vasoactive inhibitory peptide and 5hydroxytryptamine close to the vessels in the lamina propria.

We observed that GAVE was not improved by successful portal decompression following TIPS (one patient) or by endoscopic laser therapy (one patient not included in the present report) whereas the antral mucosal lesions disappeared completely after liver transplantation. This suggests that GAVE could be related to increased secretion of yet unidentified vasoactive substances in the presence of liver disease. For example, glucagon and nitric oxide secretion were found to be increased in cirrhotic patients. Local disturbances in vascular tone must be involved to explain that vascular ectasias develop specifically in the antrum.

As illustrated in this series of patients, the management of chronic bleeding from GAVE is not a simple issue. Therapeutic options include endoscopic laser therapy, oestrogenprogesterone therapy, and surgical antrectomy.

Endoscopic laser coagulation of the lesions in GAVE has been shown to induce improvement of vascular lesions as well as reduction in transfusion requirement in the majority of patients after a median of six months of treatment. $^{22} 23$ In the diffuse form of GAVE, however, results are poorer, ${ }^{24}$ probably because of the extensive area that needs to be treated with the laser probe. Medical therapy using female sex hormones has been tested in GAVE, because of their reported beneficial effects on the recurrent bleeding from gastrointestinal vascular malformations. Recurrent blood loss was successfully controlled by oestrogenprogesterone treatment in three case reports ${ }^{25-27}$ but treatment did not eradicate the antral vascular lesions.

Surgical antrectomy can cure the disease permanently. ${ }^{1628}$ However, surgery carries a significant risk of mortality in cirrhotic patients with portal hypertension. In the present series, preoperative TIPS placement made the antrectomy easier and faster by avoiding operative blood losses related to the presence of portal hypertension, but two patients nevertheless died from multiorgan failure after surgery.

In conclusion, the results of this study show that portal vein decompression does not reduce chronic bleeding from GAVE in cirrhotic patients. Although the pathophysiology of these gastric vascular lesions is not fully understood yet, portal hypertension does not seem to play a role. Portal decompression by TIPS can be useful before antrectomy to reduce the peroperative bleeding due to collaterals in cirrhotic patients with GAVE; however, the use of TIPS should be restricted to cirrhotic patients with good liver function, due to the risk of TIPS induced progressive liver failure.

The authors wish to express their gratitude to Dave Guérette and Marie-Cécile deBearn for excellent technical support and Manon Bourcier for editing the manuscript.

1 Papazian A, Braillon A, Dupas JL, et al. Portal hypertensive mucosa: an endoscopic study. Gut 1986;27:1199-203.

2 Payen JL, Cales P, Voigt JJ, et al. Severe portal hypertensive gastropathy and antral vascular ectasia are distinct entities in patients with cirrhosis. Gastroenterology 1995;108:13844.

3 Hosking SW, Kennedy HJ, Seddon I, et al. The role of propranolol in congestive gastropathy of portal hypertension. Hepatology 1987;7:437-41.

4 Perez-Ayuso RM, Pique JM, Bosch J, et al. Propranolol in prevention of recurrent bleeding from severe portal hyper-
tensive gastropathy in cirrhosis. Lancet 1991;337:1431-4.

5 Orloff MM, Orloff MS, Orloff SL, et al. Treatment of bleeding from portal hypertensive gastropathy by portacaval ing from portal hypertensive gastrop

6 Colombato L, Spahr L, Martinet J, et al. Haemodynamic adaptation two months after transjugular intrahepatic portosystemic shunt (TIPS) in cirrhotic patients. Gut 1996;39: tosystem

7 Lafortune M, Martinet JP, Denys A, et al. Short and long-term hemodynamic effects of TIPS: a doppler/ manometric study. AfR Am $\mathcal{F}$ Roentgenol 1995;164:9971002

8 Gostout CJ, Viggiano TR, Galm R. Acute gastrointestinal bleeding from portal hypertensive gastropathy: prevalence and clinical features. Am $\mathcal{F}$ Gastroenterol 1993;88:2030-3.

9 Viggiano TR, Gostout CJ. Portal hypertensive intestinal vasculopathy: a review of the clinical, endoscopic and histopathologic features. Am 7 Gastroenterol 1992;87:94454.

10 Jabbari M, Cherry R, Lough J, et al. Gastric antral vascular ectasia: the watermelon stomach. Gastroenterology 1984;87: 1165-70.

11 Suit P, Petras R, Bauer T, et al. Gastric antral vascular ectasia. A histologic and morphometric study of the "watermelon stomach". Am f Surg Pathol 1987;11:750-7. 
12 Spina PG, Arcidiacono R, Bosch J, et al. Gastric endoscopic features in portal hypertension: final report of a consensus
conference, Milan, Italy, September $19,1992.7$ Hepatol conference, Mil

13 Panes J, Bordas JM, Pique JM, et al. Increased gastric mucosal perfusion in cirrhotic patients with portal hypertensive gastropathy. Gastroenterology 1992;103:187582 .

14 Tanoue K, Hashizume M, Wada $\mathrm{H}$, et al. Effects of endoscopic injection sclerotherapy on portal hypertensive gastropathy: a prospective study. Gastrointest Endosc 1992; 38:582-5.

15 Cales P, Voigt JJ, Payen JL, et al. Diffuse vascular ectasia of the antrum, duodenum and jejunum in a patient with nodular regenerative hyperplasia. Lack of response to portosystemic shunt or gastrectomy. Gut 1993;34:558-61.

16 Gostout CJ, Viggiano TR, Ahlquist DA, et al. The clinical and endoscopic spectrum of the watermelon stomach. $\mathcal{F}$
Clin Gastroenterol 1992;15:256-63.

17 Jouanolle H, Bretagne J, Ramee $M$, et al. Antral vascular ectasia and scleroderma. Endoscopic, radiologic and anatomopathologic aspects of an uncommon association. Gastroenterd

18 Tobin R, Hackmann R, Kimmey M, et al. Bleeding from antral vascular ectasia in marrow transplant recipients. Gastrointest Endosc 1996;44:223-9.

19 Charneau J, Petit R, Cales P, et al. Antral motility in patients with cirrhosis with or without gastric antral vascular ectasia. Gut 1995;37:488-92.
20 Quintero E, Pique J, Bombi J, et al. Gastric mucosal vascular ectasias causing bleeding in cirrhosis. A distinct entity associated with hypergastrinemia and low serum levels of pepsinogen I. Gastroenterology 1987;93:1054-61.

21 Lowes J, Rode J. Neuroendocrine cell proliferation in gastric antral vascular ectasia. Gastroenterology 1989;97:207-12.

22 Gostout C, Ahlquist D, Radford C, et al. Endoscopic laser therapy for watermelon stomach. Gastroenterology 1989;96: 1462-5.

23 Tsai H, Smith J, Danesh B. Successful control of bleeding from gastric antral vascular ectasia (watermelon stomach) by laser photocoagulation. Gut 1991;32:93-4.

24 Potamiano S, Carter C, Anderson J. Endoscopic laser treatment of diffuse gastric antral vascular ectasia. Gut 1994;35: 461-3.

25 Schoonbroodt D, Horsmans Y, Hoang P, et al. Vascular gastric lesions, Crest syndrome and primary biliary cirrhosis: efficacy of oestrogen-progesterone treatment. Gastroenterol Clin Biol 1994;18:649-51.

26 Manning R. Estrogen/progesterone treatment of diffuse antral vascular ectasia. Am f Gastroenterol 1995;90:154-6.

27 Moss S, Ghosh P, Thomas D, et al. Gastric antral vascular ectasia: maintenance treatment with oestrogenprogesterone. Gut 1992;33:715-17.

28 Kurger R, Ryan M, Dickson K, et al. Diffuse vascular ectasia in the gastric antrum. Am f Gastroenterol 1987;82:4216. 